\title{
Design and Detection of Underground Cable Fault Using Raspberry Pi \& IoT System
}

\author{
Mr. M. Dinesh, Mr. K. Vairaperumal, Mr. P. Senthilkumar \\ Assistant Professor, Department of EEE, \\ Mohamed Sathak A. J. College of Engineering, Egattur, Chennai, Tamil Nadu, India
}

\begin{abstract}
This paper proposes fault location model for underground power cable using raspberry pi and the Internet of Things which is based on the internet, which means the information will be transferred through the internet access. The aim of this method is to determine the distance of underground cable fault from base station in kilometres and also find the location of that faulty place. This paper uses the simple concept of Current Transformer Theory (CT Theory). When any fault like short circuit occurs, voltage drop will vary depending on the length of fault in cable; since the current varies Current Transformer is used to calculate the varying current. The signal conditioner manipulates the change in voltage and a microcontroller is used to make the necessary calculations so that the fault distance is displayed by IOT devices. These fault details are after sent to any access point through the internet and displayed.
\end{abstract}

KEY WORDS: Current Transformer, Voltage Regulator, Raspberry Pi.

\section{INTRODUCTION}

An embedded system is special-purpose computer system designed for performing a dedicated function and program. It is a general-purpose computer, such as a personal computer, an embedded system performs a one or few pre-defined tasks, and functions usually with very specific requirements. Since the system is specially made to do a particular tasks, and functions design engineers can optimize it, reducing its size and cost price of the product.

An embedded system is a grouping of mechanical, electrical components along with a computer, hidden inside, to perform a single function devoted purpose. There are more computers designed in this planet there are people and most of the computers are singlechip microcontrollers that are the brains of an embedded system. Embedded systems are a omnipresent component of our day by day lives. We relate with hundreds of tiny computers each day that are embedded into our houses, our cars, our bridges, our toys and our work. so have the capability of the microcontrollers embedded into our devices. As a result the world needs a skilled work force to widen and deal with products based on embedded microcontrollers.

The ground-breaking aspect of this class is to effectively educate a course with a substantial lab part within the Massive Open Online Course (MOOC) format. If MOOCs are truly going to convert the education, then they must be able to convey laboratory classes. This donation will go a long way in untying the professed complication in delivering a laboratory practice to tens of thousands of students. If charming, the technique developed in this class will notably convert the MOOC situation.

\section{LITERATURE SURVEY}

1. Dhekale P.M., Bhise S.S., Deokate N.R. (2015): This paper proposes fault place model for secretive power cable using microcontroller. The main concept underground cable fault from base station in kilometres. A set of resistors are therefore used to symbolize the cable and a dc voltage is fed at one end and the fault is detect by detect the vary in voltage using analog to voltage converter and a microcontroller is used to make the necessary calculation so that the fault distance is display on the LCD display. 
2. Prof. Arjun Nichal, Mr. Sudarshan Bhosal Mr. Vaibhav Shirsavade, Mr. Yogesh Jadhav (2016): This paper propose about fault location model for underground power cable using microcontroller and the thing which is based on the internet means the in order will transfer from end to end the internet access. That is to settle on the detachment of underground cable fault from the base station in the kilometer and also locate the exact location of that broken down place.. When any fault like short circuit occur, voltage drop will vary depending on distance end to end of fault in cable, since the current varies. A set of resistor are then used to signify the cable, since the current end and the fault is detected by detect the change in the voltage using analog to voltage converter and a microcontroller is used to make the required calculation so that the fault distance is display on the LCD display. This fault details after send to any admission point through the internet.

3. Nikhil Kumar Sain, Rajesh Kajla, Mr. Vikas Kumar (2016): This paper suggest fault position model for underground power cable using microcontroller. The aim of this scheme is to conclude the distance of underground cable fault from base station in kilometers. This scheme uses the easy idea of ohm's law. When any fault like short circuit occurs, voltage drop will vary depending on the length of fault in cable, because the current varies. A set of resistors are consequently used to represent the cable and a dc voltage is fed at one end and the fault is detect by detecting the change in voltage using analog to voltage converter and a microcontroller is used to make the required calculation so that the error distance is displayed on the LCD display.

4. T. Nandhini, J. Shalini, T. Sai Sangeeetha, D. Gnanaprakasam (2017): The project is planned to detect the place of fault in underground cable lines from the base station to exact place in kilometers using an Arduino micro controller kit. In the urban areas, the electrical cable runs in undergrounds as an alternative of overhead lines.

\section{EXISTING SYSTEM}

The underground fault finding using the presented system which is fault detection using GSM only allows a exacting user to get the details about the location of the fault. Only a particular user can have the access to the facts of fault. The operating speed of
Arduino is relatively lower than the raspberry pi hence the duration between rate of fault and display of result will be more.

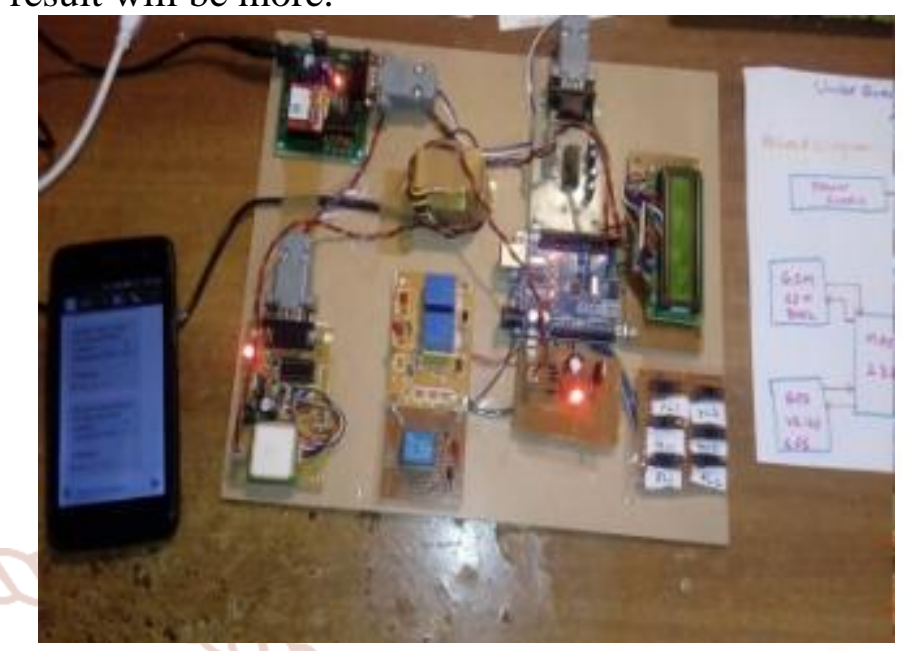

Fig. 1: Existing system Model.

\section{Block Diagram of a Proposed System}

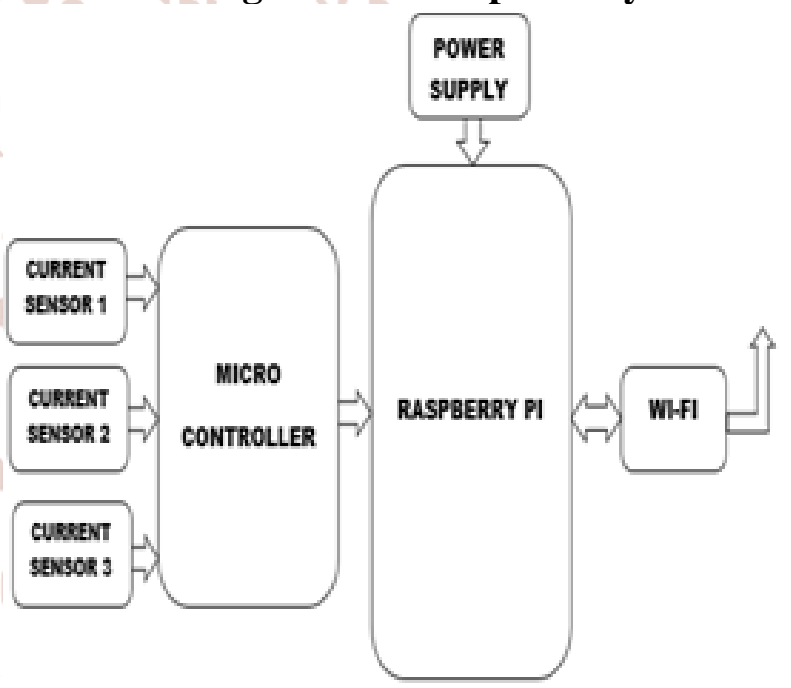

Fig. 2: Block Diagram of a Proposed System

\section{RASPBERRY PI}

Raspberry Pi has very minute size and low price. Raspberry Pi has Quad core Broadcom BCM2836 900 $\mathrm{MHz}$ CPU and 1GB RAM. It performs the effort like that of computer thus it can be referred as minicomputer. It has to be work on Python language. And it use Raspbian operational system based on Debian distribution of LINUX. In Python user don't have to openly tell the computer whether a patchy is a number or a list, or a string; the predictor figures out the data types when execute the script. Python analyst can be run in two ways: as an interactive shell to execute individual commands. Command line program to perform standalone script. Integrated development environment (IDE) bundled with Python and the Raspberry Pi is called IDLE. 
Raspberry PI organizer plays an vital role in scheming the whole system. It acts as a heart of the system which is next interfaced with triggering circuit. This interfacing connecting both circuits shares or transmit the data in the form of PWM signals to the triggering circuit.

\section{A. CPU CORE}

ARM 1176JZF-S (armv6k) 700MHz

$>$ RISC Architecture and low power draw

$>$ Not well-matched with traditional PC software

\section{B. GRAPHICS PROCESSING UNIT}

$>$ Broadcom Video IV

$>$ Specialized graphical instruction sets

\section{RAM}

512MB (Model B rev.2)

256 MB (Model A, Model B rev 1)

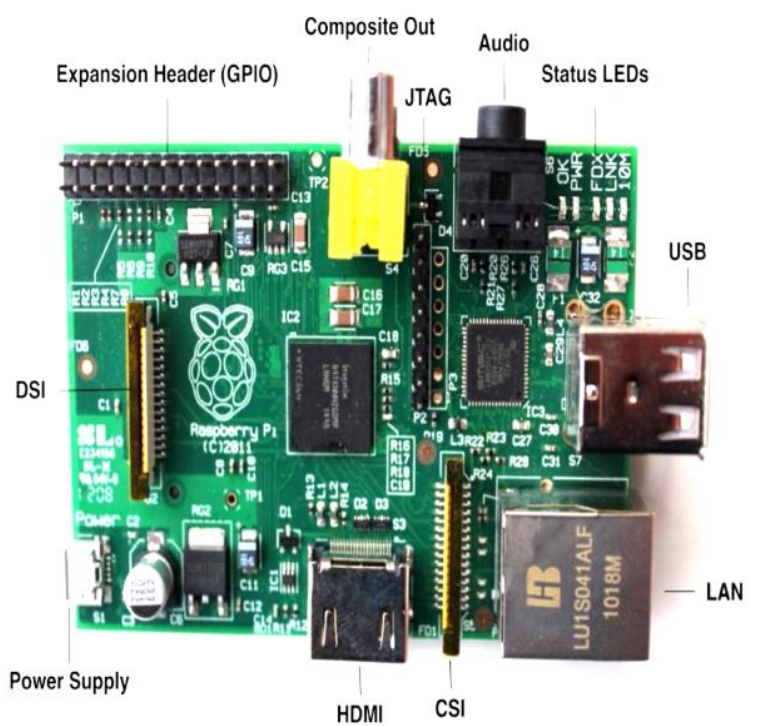

Fig. 3: Raspherry Pi Model 3Control.

\section{HARDWARE UNIT}

\section{A. POWER SUPPLY}

A power supply provides

machinery with electric power. 7805 is a $5 \mathrm{~V}$ fixed three terminal positive voltage regulators IC. The IC has features such as safe in use area protection, thermal shut down, internal current limiting which makes the IC very rugged.

Output currents up to $1 \mathrm{~A}$ can be drained from the IC provide that there is a proper heat sink. A $12 \mathrm{~V}$ transformer steps down the main voltage, 1A bridge rectifies it and capacitor $\mathrm{C} 1$ filters it and 7805 regulate it to make a steady $5 \mathrm{~V}$ DC. The crcuit schematic is given below.

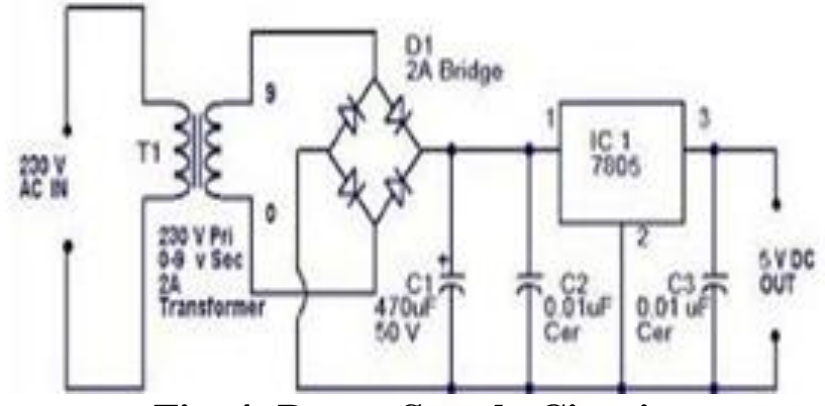

Fig. 4: Power Supply Circuit.

\section{B. CURRENT TRANSFORMER}

A current transformer (CT) is a type of transformer that is used to determine alternating current (AC). It produces a current in its secondary which is relative to the current in its primary. Current transformers, all along with voltage or potential transformers are instrument transformers. Instrument transformers scale the large principles of voltage or current too small, consistent values that are easy to handle for instrument and protective relays. The instrument transformers divide measurement or protector circuits from the high voltage of the primary system. The current transformer presents a negligible load to the primary circuit. Current transformers are the current-sensing units of the power system and are used at generating stations, electrical substations, and in industrial and commercial electric power distribution.

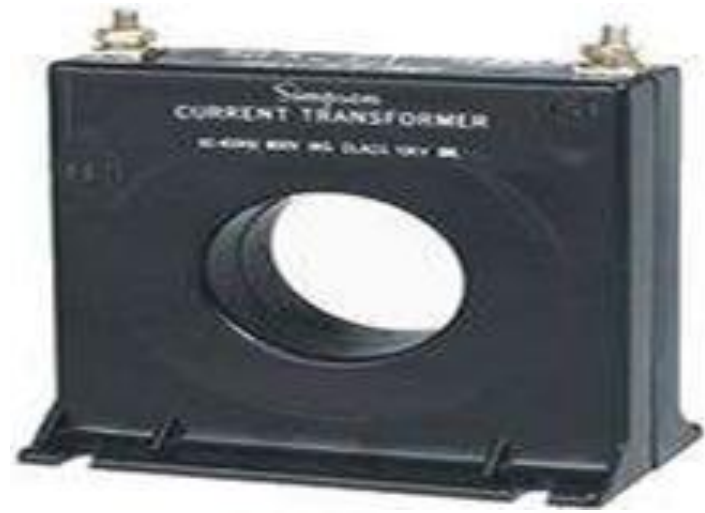

Fig. 5: Current transformer.

CTs are exacting by their current ratio from primary to secondary. The rated secondary current is normally standardized at 1 or 5 amperes. For example, a 4000:5 CT secondary winding will present an output current of 5 amperes when the primary winding current is 4000 amperes. This ratio can also be used to position the impedance or voltage on one side of the transformer, given the opposite value at the other side. For the 4000:5 CT, the secondary impedance can be found as $Z_{S}=N Z_{P}=800 Z_{P}$, and the secondary voltage can be found as $\mathrm{V}_{\mathrm{S}}=\mathrm{NV}_{\mathrm{P}}=800 \mathrm{~V}_{\mathrm{P}}$. In some 
cases, the secondary impedance is referred to the primary side, and is found as $Z_{S^{\prime}}=N^{2} Z_{P}$. Referring the impedance is done purely by multiply initial secondary impedance value by the current ratio. The secondary winding of a CT can have taps to give a range ofs ratio, five taps being common.

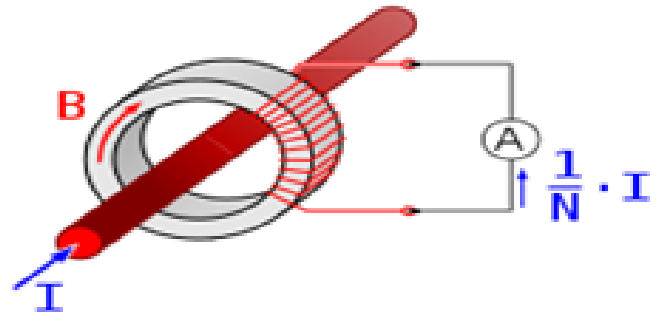

Fig. 5: Current transformer layout

\section{BRIDGE RECTIFIER}

When four diodes are related as shown in figure, the circuit is called as bridge rectifier. A bridge rectifier is an arrangement of four or more diodes in a bridge circuit arrangement which provides the same output polarity for either input polarity. It is used for convert an alternating current (AC) input into a direct current (DC) output which periodically reverses direction, to direct current (DC), which flows in only one direction. The input to the circuit is applied to the crosswise opposite corners of the network, and the output is taken from the lasting two corners. A rectifier diode lets electrical current flow in only one direction and is generally used for power supply operation. Rectifier diodes can handle top current flow than regular diodes and are normally used in order to change alternating current into direct current.

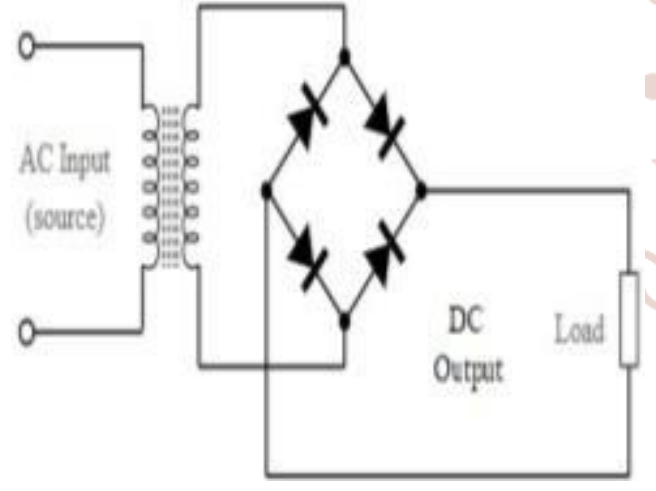

Fig. 6: Diode Bridge rectifier

\section{IC VOLTAGE REGULATORS}

Voltage regulators involve class of widely used IC's. Regulators IC units surround the circuitry for reference source, comparator amplifier, and control device and overload protection all in a single IC. IC units present regulation of either a fixed positive voltage, a fixed negative voltage, or an adjustable set voltage.
LM7805 PINOUT DIAGRAM

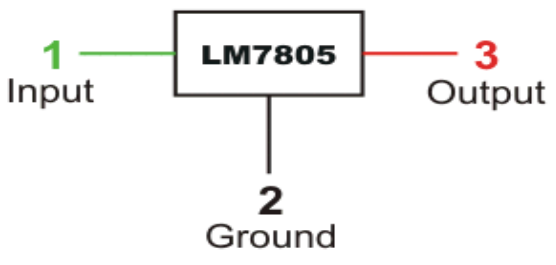

LM7805

Fig. 7: IC Voltage regulator

A fixed three-terminal voltage regulator has an unfettered dc input voltage, Vi, practical to one input terminal, a regulated dc output voltage, Vo, from a second terminal, with the third terminal connected to the ground. The series 79 regulators provide fixed negative keeping pace voltages from 5 to 24 volts. Equally, the series 79 regulators provide fixed negative regulated voltages from 5 to 24 volts.

\section{E. RASPBERRY PI PROGRAMMING}

The Raspberry $\mathrm{Pi}$ groundwork recommends the use of Raspbian, a Debian-based Linux operating system. Other third-party operating system available via the officer website include Ubuntu MATE, Windows 10 IoT Core, RISC OS and specialized distributions for the Kodi media centre and classroom management. Many added operating systems can too run on the Raspberry Pi.

\section{OTHER OPERATING SYSTEMS (NOT LINUX-} BASED)

$>$ RISC OS Pi (a special cut down version RISC OS Pico, for $16 \mathrm{MB}$ cards and larger for all model of Pi $1 \& 2$, has also been made available.)

FreeBSD

NetBSD

Windows 10 IoT Core - a no-cost edition of Windows 10 offered by Microsoft that runs natively on the Raspberry Pi 2.

\section{F. HARDWARE FLOWCHART}

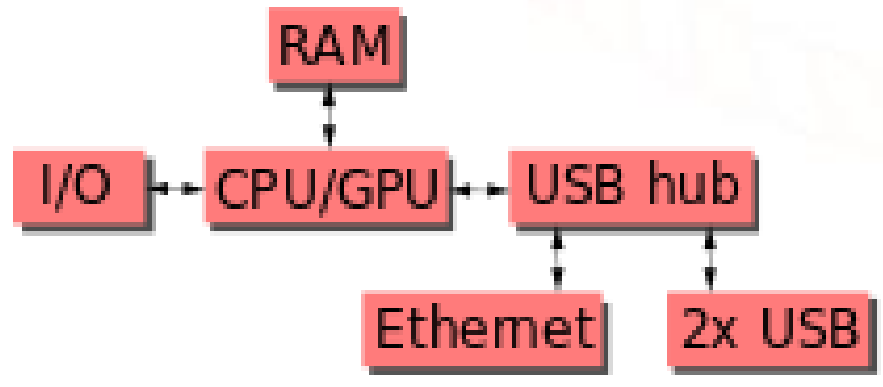

Fig. 8: Hardware Flow chart 


\section{G. INTERNET OF THINGS}

The Internet of Things (IoT) is the network of material plans which allow these objects to connect and exchange data. Each thing is exclusively identifiable from side to side its embedded computing system but is able to interoperate within the existing Internet infrastructure.

\section{HARDWARE DIAGRAM}

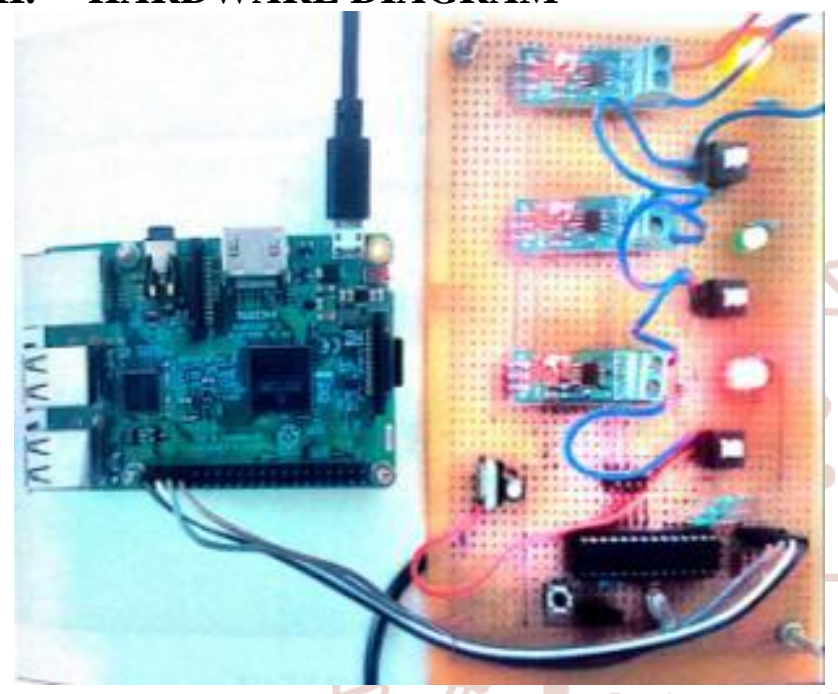

Fig. 9: project Hardware

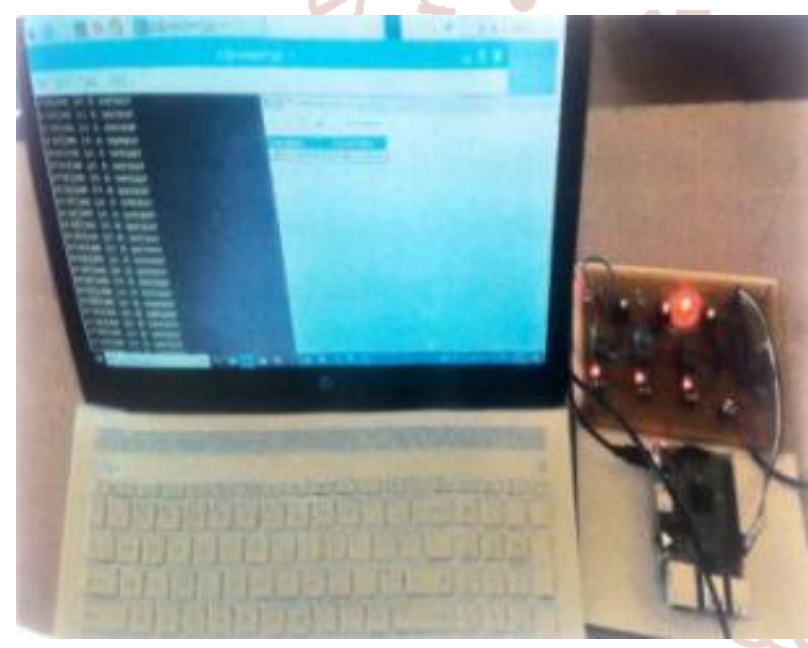

Fig. 10: Working Model Interfaced with CPU

\section{CONCLUSION}

It can accomplish advanced fault detection precision in underground cable, mainly for high impedance early faults. It can control almost the entire length of the cable. It uses the effortless conception of Current Transformer Theory so the fault can be easily detected and repaired. It detects the exact spot of short circuit fault in the underground cable from feeder end. The dispensation speed is higher so the time delay between the detection of fault and display of the result is lower. The data can be access from anyplace by means of cloud storage.

\section{REFERENCES}

1. T. Nandhini1, J. Shalini2, T. Sai Sangeeetha3, D. Gnanaprakasam4 Student1, 2. Nikhil Kumar Sain, Rajesh Kajla, Mr. Vikas Kumar "A New Approach to Underground Cable Fault Distance Conveyed Over GSM “, IEEE Journal on Power Delivery e-ISSN: 2278-1676,p-ISSN: 2320-3331 Volume 11, Issue 2 Ver. III (2016).

2. Prof. Arjun Nichal, Mr. Sudarshan Bhosale, Mr. Vaibhav Shirsavade, Mr. Yogesh Jadhav Assistant Professor, "IOT Based Underground Wire Fault Detection \& Technique" Electronics \& Telecommunication Department, AITRC, Vita, India 1 Student (2016).

3. Sahana S ,2Harish Kumar B M, 3Anu S M 4 Vani $\mathrm{H}$ V, 5Sudha T, 6Prashanth Kumar H K 1,2,3 U. G Student, 4,5,6 Associate Professor, "Analysis of fault detection and its location using microcontroller for underground cables" Department of EEE, SJMIT, Chitradurga, Karnataka, INDIA.

4. Raj Kamal Kakoti2 Assistant Professor, "Underground Cable Fault Locator Manish Paul1" , Electrical Engineering Dept, Girijananda Chowdhury Institute of Management and Technology, Guwahati, Assam, India1,

5. ShirtarReshma*,Gaykar Monika, Parve Ashwini, TambeSaurabhi, Chaudhari S. S., "Underground Cable Fault Distance Locator By Using Microcontroller" Department of Electronics \& Telecommunication Engineering, Jaihind Polytechnic Kuran, India.

6. D. A.Shaikh1, Dhamale Amar P 2, Gulve Mayur S3 , Supekar Suraj D4 1,2,3,4 "Remote Fault Finding in Underground Cables Using Wireless Communication" Department of Electronics And Telecommunication Engineering, (India). 